\title{
Continence promotion for older hospital patients following surgery for fractured neck of femur: Pilot of a randomized controlled trial
}

\author{
Lynne Parkinson ${ }^{1,2}$ \\ Pauline Chiarelli ${ }^{3}$ \\ Jennifer Byrne' \\ Richard Gibson' \\ Suzanne McNeill ${ }^{4}$ \\ Gillian Lloyd ${ }^{5}$ \\ Wendy Watts ${ }^{6}$ \\ Julie Byles'
}

'Research Centre for Gender, Health and Ageing, Faculty of Health, The University of Newcastle, NSW, Australia; ${ }^{2}$ Hunter Ageing Research, Faculty of Health, The University of Newcastle, NSW, Australia; ${ }^{3}$ Faculty of Health, The University of Newcastle, NSW, Australia; ${ }^{4} \mathrm{NC}$ Trauma Orthopedics, John Hunter Hospital, New Lambton Heights, NSW, Australia; ${ }^{5}$ Hunter New England Health, Wallsend Community Health Centre, Wallsend, NSW, Australia; ${ }^{6}$ Royal Newcastle Centre, John Hunter Hospital, New Lambton Heights, NSW, Australia
Correspondence: Lynne Parkinson RCGHA, Level 2, David Maddison Building, University of Newcastle, Callaghan, NSW 2308, Australia

$\mathrm{Tel}+61249138232$

Fax +612492138323

Email lynne.parkinson@newcastle.edu.au
Abstract: Evidence suggests that bladder control problems develop or worsen as a result of fractured neck of femur (\#NOF) and its subsequent management.

The primary aim of this study was to reduce the prevalence and severity of post surgery continence problems among patients, aged from 60-years, undergoing surgery for \#NOF, using a best practice "case-management model" multifactorial intervention.

Eligible consenting patients admitted with \#NOF were randomized to intervention or control group. Self-report questionnaires compared pre-surgery, post surgery, and follow-up continence status between groups.

This pilot randomized controlled trial, which included 45 eligible patients aged 60 to 93-years, found no evidence that the intervention was effective in reducing prevalence of post-surgery incontinence in this acute setting. Staff surveys highlighted the need for open communication between the research team and hospital staff. Unclear results were attributed to the small sample size.

A central outcome was evidence that intervention to improve continence management for older people post-surgery is imperative. Focused assessment and treatment for those most at risk of incontinence after \#NOF would be more acceptable to staff and a more efficient use of resources. A simple screening tool would ensure that those most at risk are detected, and targeted for care.

Keywords: urinary incontinence, prevention, management, fractured neck of femur, hip surgery, randomized controlled trial, elderly

\section{Introduction}

Incontinence is common among older people (Millard 1996), morbid (Fonda 1986), progressive (Grimby et al 1993), costly (Ouslander 1990; Doran et al 2001), and a major factor leading to placement in nursing homes (Smith 1998).

While the prevalence of fractured neck of femur (\#NOF) is decreasing in Australia, the number of hip fractures being treated is increasing due to the increasing proportion of older people within the population (Boufous et al 2004). Admission to high dependency care units following surgery for \#NOF is common (AHCPR 1996), and urinary incontinence is known to be a contributing factor to decisions related to these admissions (Thom et al 1997). One study has estimated that $50 \%$ of residents in Australian nursing homes are "wet" (Millard 1996).

Short and long term outcomes following surgery for \#NOF have been well studied (Sanders 1999), with the exception of bladder function. Available evidence suggests that bladder control problems develop or worsen as a result of \#NOF and its subsequent management (Palmer et al 1997; Halm et al 2003), but there is little information available regarding other changes in lower urinary tract symptoms (LUTS). 
Change in continence status after surgery has been associated with a number of contributing factors including medications (Ringdal et al 2003), anesthetics (Dray 1988), urinary retention (Meigs et al 1999), catheterization (Skelly et al 1992), subsequent urinary tract infection (Johansson et al 2002), and constipation (Palmer et al 1997). A recent epidemiological study (Chiarelli et al 2006), conducted with 74 eligible patients (55 at follow-up) measured the prevalence of pre-fracture and post operative bladder control problems in older people admitted with \#NOF. Surgery for \#NOF was found to be associated with significant changes in lower urinary tract function and compromised mobility with significant impact on toileting. While changes in continence status improved between two surveys conducted five days post surgery and 12-weeks post-surgery, complete resolution or return of symptoms to pre-fracture levels of bladder control did not occur (Chiarelli et al 2006).

The incidence of \#NOF is expected to double in the next 20 -years (Sanders et al 1999). Consequently, the need for interventions to improve bladder management following surgery is compelling (March et al 1996). There has been considerable work done on prevention, treatment and rehabilitation of \#NOF (March et al 1996), but few studies have included urinary incontinence as a measured outcome (Chiarelli et al 2006) and there is no past research which has explored the best ways to intervene for continence promotion. Incontinence prevention and management is problematic as it is necessarily a multidisciplinary problem, where bladder problems can be linked to many exacerbations and causes. An intervention for continence promotion must also necessarily be multifactorial, given these diverse contributions. Otherwise, responsibility for detection and management of problems can be easily overlooked.

Clinical Pathways have been shown to be effective in standardizing care and improving outcomes (Kitchiner and Bundred 1999). An evidence-based Clinical Pathway for bladder management was developed by clinicians within the Hunter in 2000 (Watts et al 2002), but not successfully implemented within the acute care setting. This research team provided the impetus for operationalizing this model pathway with the pilot work described here. Clinical Pathways are only useful if they are used consistently and if variances to the standard pathway are reliably recorded (NHS Centre for Reviews and Dissemination 1999). Effective adoption of a pathway requires the cooperation of staff across disciplines, so must be supported by significant educational and motivational effort. A major objective of this study was to change current clinical practice in regard to continence management for \#NOF patients.
Written health promotion information has been argued as acceptable and beneficial for elderly patients and their carers (Petterson et al 1994). Written resources can be particularly useful for those patients who have difficulty remembering information, those who would like a resource to refer to, and those who need details of how to seek further assistance if required. Targeted resources tailored to the specific group of interest are known to be most effective (Doak et al 1998; Kreuter et al 2000; Ryan and Lauver 2002). A specific objective of this project was to develop a tailored continence resource for patients undergoing \#NOF as one of the intervention factors.

The primary aim of this study was to reduce the prevalence and severity of post surgery continence problems among patients, aged 60-years and over, undergoing surgery for \#NOF in a large, non-metropolitan, tertiary referral, teaching hospital in Australia, by implementing a multifactorial intervention (which included promoting the use of a local Clinical Pathway, education and motivation sessions across the multi-disciplinary provider team, and a continence information booklet specific to this patient group).

\section{Method \\ Design}

A randomized controlled trial design was used with participants individually randomized to intervention or control group.

\section{Sample and setting}

The sample included men and women aged 60-years and over who were admitted to a large, non-metropolitan, tertiary referral, teaching hospital in NSW, Australia, from September 2005 to February 2006. The study hospital had the highest patient admissions for hip fracture in NSW in 2003-2004 (HSRG 2006). Patients who did not have the cognitive capacity to consent to the study (Mini Mental State Examination (Folstein et al 1975) score of less than 24, confused or delirious), did not speak English sufficiently well to understand study requirements, were deemed too ill to participate by nursing staff, or had in-dwelling catheters, were excluded.

\section{Procedure}

Ethical Approval was obtained from Hunter New England Area Health Service and The University of Newcastle Human Research Ethics Committees. Table 1 summarizes the study protocol.

Eligible patients who told staff (Nurse Unit Manager [NUM] or Clinical Nurse Consultant [CNC]) they would talk 
Table I Flow chart of study protocol

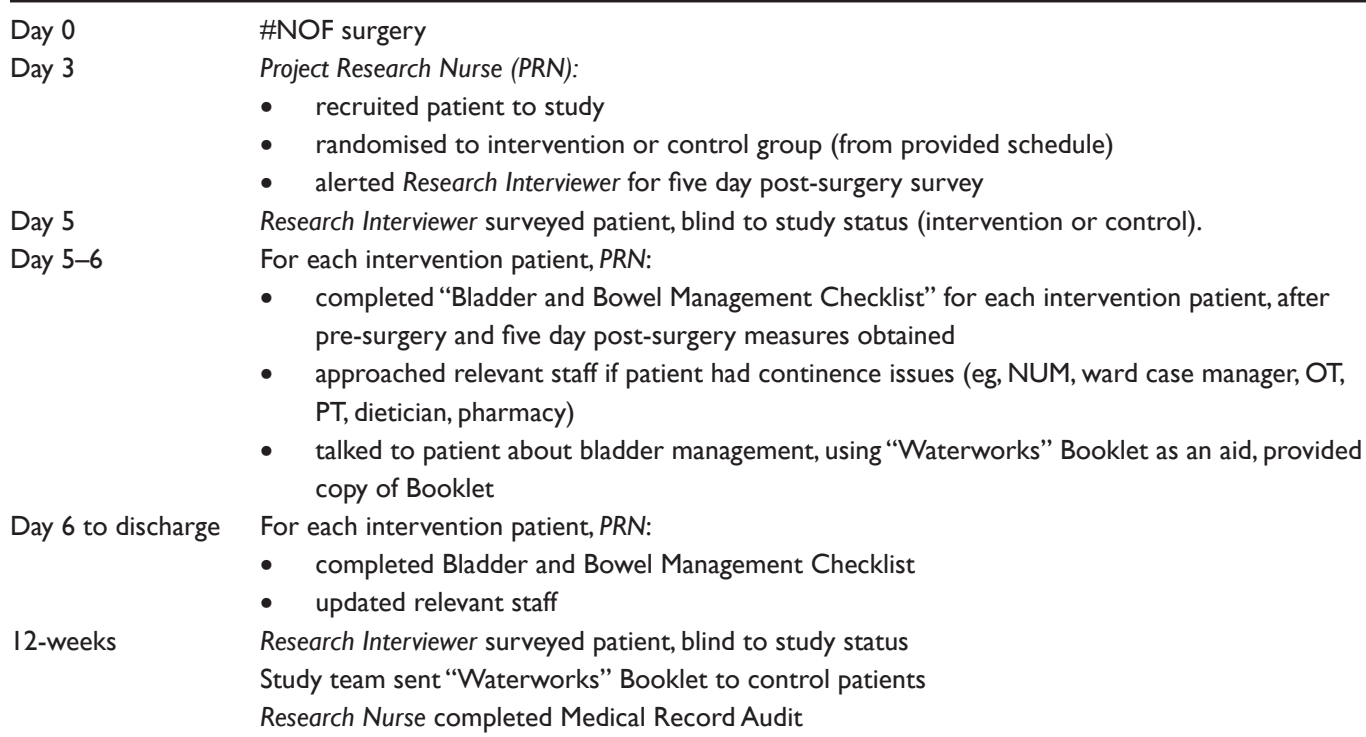

to the Project Research Nurse (PRN) had the study explained to them by the PRN on the third post-operative day, and were given an information sheet and a consent form. This time frame was chosen to allow for post-operative recovery.

Blinded random allocation of consenting eligible patients was achieved by using sealed envelopes marked with consecutive IDs to conceal study status. Once an ID was allocated, the study status was checked. All consenting participants were approached by a Research Interviewer on the fifth post-operative day, and administered the five day post-surgery survey.

The intervention strategies (detailed below) were implemented with all intervention patients. The control group received normal care.

Twelve weeks after surgery, a Research Interviewer (blind to patient study status) contacted control and intervention participants to arrange a telephone follow-up survey.

At approximately 12-weeks post-surgery, a trained research nurse undertook a Patient Chart Audit for all participants.

Staff surveys were administered twice over the intervention period - at implementation, and at four months. Staff surveyed included NUMs, Occupational Therapists, CNCs and Physiotherapists. When face to face appointments were not possible, staff were emailed the survey to complete and return by email to the Project Manager.

\section{Intervention}

With input from the multi-disciplinary clinical team, the intervention developed as a multifactorial best practice "case-management model". The main components of the intervention were:

1. Implementation of an evidence-based collaboratively developed Clinical Pathway for Bladder Management after \#NOF.

The Clinical Pathway was finalized by a working group of CNCs (Continence and Orthopaedic) following a half-day multi-disciplinary workshop with hospital staff to identify best practice bladder care for patients. The Clinical Pathway included measures to reduce the likelihood of problems, and focused on using evidencebased continence management practices (Watts et al 2002), such as, early removal of catheters, prevention of dehydration and constipation, facilitation of toileting, and identification, investigation and management of bladder symptoms.

The intervention had a core strategy of employment of an experienced Project Research Nurse (PRN) whose role was to monitor intervention patient continence care and outcomes and ensure action and, where appropriate, referral to the multidisciplinary team (a range of professionals already involved in the patient's care).

A Bladder and Bowel Management Checklist (B and B Checklist) was developed to operationalize the Clinical Pathway. All intervention patients were monitored each day by the PRN using the B and B Checklist, which assessed patient status across domains relevant to maintaining continence. The PRN liaised with the Case Manager or NUM on the ward to discuss problems evident from the B and B Checklist and advocate action. 
2. Staff awareness raising sessions across the multidisciplinary provider team, within the hospital and community health settings.

Staff awareness raising included several strategies. Two multi-disciplinary workshops (including nurses, physiotherapists, OT's, medical practitioners, pharmacists, and dietitians) were conducted to ensure that staff across the clinical team were aware of the importance of continence to patient outcomes and were primed to respond to continence management issues for intervention patients.

Ongoing in-service sessions were conducted with nursing staff, allied health personnel and surgical registrars to alert staff to best practice bladder care following \#NOF, to facilitate their response to the Clinical Pathway and the prompts from the PRN for intervention patients, and to update staff on emerging study data. Two Newsletters were circulated to all relevant hospital staff, via email, throughout the study, detailing the aims of the project and providing information on study progress. In regular "Meet and Greet" sessions, the PRN visited all relevant wards to ensure that they and the study were readily recognised by staff, and to keep staff in touch with the study.

3. Provision of an information booklet "Waterworks after Hip Surgery"

A "Waterworks after Hip Surgery" booklet was developed for this pilot, and tested with focus groups of patients to ensure relevance to the target group. The booklet was designed to be given to patients while still in hospital, where they would be able to ask questions about the contents. The PRN explained the contents of the booklet to each intervention patient and provided contact information for a local community continence advisor (eg, continence nurse, community nurse, OT, physiotherapist) should the patient need assistance postdischarge. This booklet was sent to control patients after the 12-week interview.

4. Provision of female urinary bottle for appropriate patients

The intervention group also had access to female urinary bottles, introduced when early results from the five day post surgery survey showed that women reported urgency to urinate when a bed pan was unavailable. Nursing staff or the PRN offered the bottle, explained its use and let patients decide if they would like to trial it. If the patient trialed the bottle, they were asked some questions about ease of and satisfaction with use. The bottle was not offered to very frail patients or to those who also had arm fractures.
5. Follow-up within the Community Health Services where appropriate.

The Waterworks Booklet provided a local contact for patients who needed continence advice. If necessary, at discharge, Continence $\mathrm{CNC}$ referral was recommended for further intervention and/or follow-up in the home setting.

\section{Measures}

\section{The five-day survey}

The five-day survey included questions about bladder control, symptoms (such as urinary incontinence, bladder urgency, nocturia), symptom frequency and severity, contributing co-morbidities, and drugs known to promote urinary incontinence or urinary urgency (Hald et al 1991; Donovan et al 1996; Gunthorpe et al 2000), before and after surgery. The continence measures were adapted from the DANPSS (Donovan et al 1996), chosen for its brevity and therefore appropriateness to the acute care setting.

Participants were asked to report their continence symptoms on a four point scale, ranging from "No" through to "Every time", and the extent to which the symptom was a problem, on a four point scale ranging from "Not a problem" to a "Serious problem". These responses were dichotomized, where participants reporting "Daily" or "Every time" (as opposed to "Never" or "Hardly ever") were classified as having the symptom, while those who reported the symptom as being "Quite a problem" or "A serious problem" were classified as experiencing a problem. Symptoms were grouped into three categories: storage, irritative and voiding.

\section{The I2-week survey}

The 12-week survey contained the same questions as asked at five days post surgery, but for the "last month" timeframe, to determine if continence status had changed.

\section{Patient Chart Audit}

The Chart Audit provided details of date of birth, sex, education, date of admission, index condition and co-morbidities (scored using the Charlson index) (Charlson et al 1987), date and status at discharge, length of time before arrival at hospital, length of time of surgical procedure, surgical anesthesia, post-operative pain management, urinary retention and management strategies used, symptoms or diagnosis of urinary tract infections, and medications. The Chart Audit also identified incontinence interventions occurring during the patient's hospital stay and those recommended on discharge. 


\section{Staff surveys}

Staff surveys asked about knowledge and understanding of the study, and changes to clinical practice in response to the study.

\section{Bladder and Bowel Management Checklist ( $\mathrm{B}$ and $\mathrm{B}$ Checklist)}

The B and B Checklist (described above) was also used as a process measure of staff intervention activity.

\section{Analyses}

The main outcome measure was incontinence severity after surgery. It was hypothesized that the intervention would reduce the prevalence of incontinence by $10 \%$ at follow-up.

Incontinence Score was calculated from self reported patient bladder function information across twelve bladder related symptoms. Each of the twelve symptoms had an associated question that asked how much of a problem this symptom posed on a daily basis. Incontinence Score was the sum of the products of the frequency of the symptom and how much of a reported problem it was (Hald et al 1991).

Severity Index is a simple index that allows urinary incontinence to be studied in epidemiological studies (Sandvik et al 1993), and is created by multiplying the reported frequency of leakage by the amount of leakage. The result is then categorised into: "None" (0), "Slight" (1-2), "Moderate" (3-4) and "Severe" (6-8).

Continence and symptom measures at five days and 12-weeks post surgery were compared to pre surgery for both intervention and control groups. Since participant numbers were small, hypothesis tests were only applied to Severity Index and Incontinence Score, which provided more comprehensive and detailed markers of continence status within one variable. Chi Squared tests were used to examine differences in scores between groups at pre-surgery, five days post surgery, and 12-week follow-up.

Descriptive analysis was undertaken for the Chart Audit and the B and B Checklist. Qualitative analyses were used to interpret responses to the Staff surveys.

\section{Results}

\section{Participants}

From September 2005 to February 2006, 173 patients underwent surgery for \#NOF at the study hospital. Despite a high consent rate (92\%), only $30 \%$ of those admitted to hospital for surgery for \#NOF were eligible for inclusion in the study. Primary reasons for ineligibility were medical (36\%) and cognitive (45\%) problems. Four patients declined to participate.
Three of the 48 consenting patients did not participate in the five day post surgery survey (one was transferred, one was too ill, and one withdrew consent). Consequently, there were 45 participants randomly allocated to intervention $(n=26)$ or control $(n=19)$ treatments.

Furthermore, 11 (7 control and 4 intervention) people were lost to follow-up at 12-weeks ( 5 were too ill, 2 died, 2 could not be contacted, 2 withdrew consent). Thus 34 participants (intervention $=15$; control $=19$ ) were included in the final analyses.

\section{Participant characteristics}

The majority of respondents were women. In the intervention group, 9 were women and 6 were men, and in the control group, 15 were women and 4 were men. In the intervention group, the mean and median age were 81-years and 82-years respectively, with an age range of 60-91-years. The mean and the median age of the control group were 81-years and 83-years respectively, with an age range of 66-91-years. There were no significant differences between treatment groups with respect to gender $(\mathrm{p}=0.2)$, age, or pre-existing conditions of stroke $(p=0.8)$, Parkinson's disease $(p=0.4)$, diabetes $(\mathrm{p}=0.2)$ and hypertension $(\mathrm{p}=0.2)$.

\section{Incontinence symptoms}

As can be seen in Table 2, urgency was reported as the primary irritative symptom by both the intervention and control groups. There were no significant differences between groups, at any time, for reported irritative symptoms. Voiding symptoms were reported by few participants. While $47 \%$ $(n=7)$ of the intervention group and $32 \%(n=6)$ of the control group and reported nocturia pre-surgery, this difference was not significant $(p=0.4)$. Due to the small sample size, apparent percentage differences often only meant one or two participants.

\section{Problematic bladder symptoms}

Table 3 shows the number of participants who reported bladder symptoms as a "problem" (those who classified the symptom as being "Quite a problem" or "A serious problem"). More commonly reported problematic symptoms were night time frequency, leakage on the way to the toilet and urgency. Again, the numbers were small and differences, in the main, represented only one or two participants in each group.

\section{Incontinence score}

Incontinence scores, as detailed in Table 4, indicated that participants were equally likely to increase, decrease or 
Table 2 Self-reported incontinence symptoms, pre-surgery, post-surgery and at follow-up, according to treatment group

\begin{tabular}{|c|c|c|c|c|c|c|}
\hline \multirow[t]{2}{*}{ Storage symptoms } & \multicolumn{3}{|c|}{ Control $(\mathrm{N}=19)$} & \multicolumn{3}{|c|}{ Intervention $(\mathrm{N}=15)$} \\
\hline & $\begin{array}{l}\text { Pre } \\
\text { N }\end{array}$ & $\begin{array}{l}\text { Post } \\
\text { N }\end{array}$ & $\begin{array}{l}\text { Follow- } \\
\text { up } \\
\mathbf{N}\end{array}$ & $\begin{array}{l}\text { Pre } \\
\text { N }\end{array}$ & $\begin{array}{l}\text { Post } \\
\text { N }\end{array}$ & $\begin{array}{l}\text { Follow- } \\
\text { up } \\
\mathbf{N}\end{array}$ \\
\hline Daytime frequency & I & I & 0 & 2 & 2 & 1 \\
\hline Night time frequency & 6 & 7 & 5 & 7 & 4 & 7 \\
\hline $\begin{array}{l}\text { Leakage on way } \\
\text { to toilet }\end{array}$ & 1 & I & 0 & 3 & 3 & 2 \\
\hline $\begin{array}{l}\text { Leakage physical } \\
\text { activity }\end{array}$ & I & 0 & 0 & 2 & 3 & 1 \\
\hline Leakage unprompted & 0 & 0 & 0 & 2 & 3 & 2 \\
\hline Irritative symptoms & Pre & Post & $\begin{array}{l}\text { Follow- } \\
\text { up }\end{array}$ & Pre & Post & $\begin{array}{l}\text { Follow- } \\
\text { up }\end{array}$ \\
\hline Burning stinging & 0 & I & 0 & 0 & I & 0 \\
\hline Urgency & 5 & 6 & 2 & 3 & 3 & 6 \\
\hline Bladder pain & 0 & 0 & 0 & 0 & 0 & 0 \\
\hline \multicolumn{7}{|l|}{ Voiding symptoms } \\
\hline Faltering stream & 0 & 2 & 0 & 3 & I & 4 \\
\hline Weak stream & 0 & 0 & 0 & I & 0 & 3 \\
\hline Initiation & I & I & 0 & I & 0 & I \\
\hline Retention & 2 & 2 & I & 2 & 0 & 1 \\
\hline
\end{tabular}

maintain scores from pre to post surgery. By 12-week follow-up the tendency was for participants' scores to be either equal to five days post surgery or one point lower.

\section{Severity index}

A shown in Table 4, before surgery, 36\% (5) of the intervention group and $63 \%$ (12) of the control group had a

Table 3 Self-report of problematic bladder symptoms, pre-surgery, post-surgery and at follow-up, according to treatment group

\begin{tabular}{|c|c|c|c|c|c|c|}
\hline & \multicolumn{3}{|c|}{ Control } & \multicolumn{3}{|c|}{ Intervention } \\
\hline & $\begin{array}{l}\text { Pre } \\
\text { N }\end{array}$ & $\begin{array}{l}\text { Post } \\
\text { N }\end{array}$ & $\begin{array}{l}\text { Follow- } \\
\text { up } \\
\mathbf{N}\end{array}$ & $\begin{array}{l}\text { Pre } \\
\mathbf{N}\end{array}$ & $\begin{array}{l}\text { Post } \\
\text { N }\end{array}$ & $\begin{array}{l}\text { Follow- } \\
\text { up } \\
\mathbf{N}\end{array}$ \\
\hline Day time frequency & 2 & 2 & 0 & 2 & 3 & 2 \\
\hline Night time frequency & 3 & 5 & I & 2 & 4 & 2 \\
\hline Leakage on way to toilet & 3 & 3 & 0 & 2 & 3 & 2 \\
\hline $\begin{array}{l}\text { Leakage with physical } \\
\text { activity etc. }\end{array}$ & 0 & 0 & 0 & I & 2 & I \\
\hline Leakage unprompted & 0 & 0 & 0 & 2 & 3 & 2 \\
\hline Burning-stinging & 0 & 1 & 0 & 0 & 0 & 0 \\
\hline Urgency & 3 & 2 & I & 2 & 4 & 2 \\
\hline Faltering stream & 0 & 0 & 0 & 0 & 0 & I \\
\hline Weak stream & 0 & 0 & 0 & I & 1 & 2 \\
\hline
\end{tabular}

level of severity of 'none'. There were some small changes between pre-surgery and 12-week follow-up with both groups showing a small increase in the numbers considered to have 'none' or a 'slight' problem.

\section{Hypothesis tests}

The results (See Table 4) of the hypothesis tests showed no statistically significant differences between groups at either pre-surgery or the 12-week follow-up.

\section{Patient Chart Audit}

The main findings from the chart audit were that there was very little difference in hospital care between the intervention and control groups, and there were many inpatient bladder care actions that could be improved. Whilst mean duration of catheterization of 64 hours (intervention) and 68 hours (control) were very similar, the median scores varied at 53.5 hours (intervention) and 64.5 hours (control), although this difference was not significant $(\mathrm{p}=0.5)$. Very few continence expert consultations were arranged for either group and discharge bladder management and referrals were not well documented in the notes. There were no significant differences between groups for time from ER to operation $(p=0.4)$, time from fall to operation $(p=0.5)$, type of surgical procedure $(p=0.8)$ or type of anesthesia $(p=0.4)$.

Table 4 Summary of incontinence scores and severity indices and hypothesis testing on these indicators, according to time period and treatment group

\begin{tabular}{|c|c|c|c|c|c|c|}
\hline \multirow{2}{*}{$\begin{array}{l}\text { Incontinence } \\
\text { Score }\end{array}$} & \multicolumn{3}{|c|}{ Control } & \multicolumn{3}{|c|}{ Intervention } \\
\hline & Pre & Post & $\begin{array}{l}\text { Follow- } \\
\text { up }\end{array}$ & Pre & Post & $\begin{array}{l}\text { Follow- } \\
\text { up }\end{array}$ \\
\hline $\mathrm{N}$ & 19 & 19 & 19 & 15 & 15 & 15 \\
\hline Mean & 3.7 & 4.6 & 0.7 & 7.1 & 7.8 & 5.9 \\
\hline Median & 0 & I & 0 & I & 2 & 0 \\
\hline Range & $(0-18)$ & $(0-22)$ & $(0-5)$ & $(0-46)$ & $(0-40)$ & $(0-50)$ \\
\hline Incontinence & Pre & Post & Follow- & Pre & Post & Follow- \\
\hline Severity & $\mathbf{N}$ & $\mathbf{N}$ & up & $\mathbf{N}$ & $\mathbf{N}$ & up \\
\hline Index & & & $\mathbf{N}$ & & & $\mathbf{N}$ \\
\hline None & 12 & 14 & 14 & 5 & 8 & 7 \\
\hline Slight & 2 & I & 3 & 3 & I & 4 \\
\hline Moderate & 3 & 2 & I & 4 & I & 3 \\
\hline Severe & 2 & I & I & 2 & 4 & I \\
\hline Total & 19 & 18 & 19 & 14 & 14 & 15 \\
\hline
\end{tabular}

Hypothesis test Pre-surgery differences Follow-up differences

Severity Index $\quad P=0.4 \quad P=0.2$

Continence Score $P=0.3 \quad P=0.3$ 


\section{$B$ and B Checklist}

Selected items from the B and B Checklist are presented in Table 5. Of the 21 participants in the intervention group, all were assessed using the checklist at least once and 14 were assessed 3 or more times. Overall, $43 \%$ experienced urinary incontinence at some time during their hospital stay, $90 \%$ were reported as having had a fluid balance chart commenced, and $100 \%$ were reported as receiving adequate fluids. Only $38 \%$ received a special diet and $35 \%$ received a dietitian referral during their hospital stay.

\section{Waterworks booklet}

Of 15 intervention participants contacted at 12-week follow-up, 10 reported they had received the booklet while in hospital. Two of the 10 requested that another booklet be sent to them despite already receiving one. Six reported that the booklet was explained by the nurse, 7 reported reading it (all of these reported that the booklet was useful), and 3 participants reported trying the pelvic floor muscle exercises. One control group participant said they received a booklet while in hospital.

\section{Female urinary bottle}

Of the 10 patients offered the female urinary bottle, one person said it was very useful and that she would use it again if needed. One patient who tried the bottle had problems with leakage. The other patients were unable to use the bottle due to frailty or medical issues.

\section{Staff surveys}

Eight staff responded to the First Staff Survey and four to the Second Staff Survey. Lack of communication and knowledge of the research project were key issues raised by staff, primarily related to high staff turnover within the hospital. Barriers to successful project implementation were identified as pressures on staff, short patient stays and project communication. A major issue was that those interviewed were more likely to see their role as "raising awareness", "facilitating" and "supporting" rather than actively participating to improve continence. Those involved in direct care saw continence promotion as secondary to their primary role, at best, or, more usually, "not a priority" and "not important".

\section{Discussion}

The primary aim of this project was to reduce the prevalence and severity of post surgery continence problems among patients, aged 60-years and over, undergoing surgery for \#NOF in a large, non-metropolitan, tertiary referral,
Table 5 Selected items from Bladder and Bowel Management Checklist

\begin{tabular}{ll}
\hline Item & $\begin{array}{l}\text { Number of patients } \\
(\mathbf{N}=\mathbf{2 1})\end{array}$ \\
\hline Number of times B and B Checklist completed & \\
$\mathrm{I}-2$ & 7 \\
3 or more & 14 \\
Ever experienced urinary incontinence & 9 \\
Accurate fluid balance chart started & 19 \\
Ever had adequate fluids & 21 \\
Ever had special diet & 8 \\
Ever had dietitian referral & 7 \\
\hline
\end{tabular}

Australian teaching hospital. This pilot randomized controlled trial, which included 45 eligible patients aged between 60 and 93-years, found no evidence that the multifactorial best practice intervention was effective in reducing the prevalence of post-surgery incontinence in this acute hospital setting.

\section{Issues and challenges}

This well planned pilot had every expectation of achieving success. An experienced team developed a robust design, utilized already tested protocols for recruitment and evaluation, implemented a locally developed Clinical Pathway as the focus of the intervention, and appeared at outset to have good clinical support (much work was done to encourage clinicians to come on board). However, there were several challenges which meant that this expectation was not met. The two main issues were slow recruitment rate and the culture of the setting itself.

The study hospital consistently has the highest number of \#NOF admissions in NSW (HSRG 2006), but, despite the hospital recording the expected patient numbers throughout during the study timeframe, and the study achieving a high consent rate, the frailty of the target population delivered an unexpectedly small sample. Illness and other factors reduced the sample size further at follow-up. Further, there appeared to be an attrition bias for the intervention group (with $42 \%$ lost to follow-up), despite careful blinded allocation to treatment. Due to the small sample size, it was not possible to meaningfully interpret changes in reporting of symptoms and apparent percentage differences often meant only one or two participants.

The involvement of Hospital staff was essential to the successful implementation of the intervention. The project had two main agents for Clinical Pathway implementation: a dedicated Project Research Nurse (PRN), and the B and B Checklist (to operationalize the Clinical Pathway) which was 
used by the PRN to monitor patients and prompt Hospital staff with recommended actions for continence management. While the core team of research personnel involved in the project remained focused and motivated, "on the ground" hospital staff experienced the usual day to day hospital workload, time pressures and constraints. The First Staff Survey highlighted the need for better communication between staff on Wards and the Research team. At this time, several strategies were implemented to attempt to increase the dose of the intervention: more staff in-services; the introduction of the female urinary bottle; "thank you" baskets for Hospital staff involved in the study to boost morale and show appreciation for participation; and, Newsletters for all staff delivered by email and by hard copy during "Meet and Greet" visits. The Staff Survey also highlighted the difficulty in encouraging clinical staff to see continence promotion as an important part of their acute care role, and, as the Patient Chart Audit indicated, the Clinical Pathway was not as fully implemented as needed.

\section{Main study findings}

Among the 34 patients followed to 12-weeks post surgery, there were no significant differences between the intervention and control group Incontinence or Severity Index scores, either at pre-surgery or at 12-weeks post surgery. The level of intervention implementation had no measurable effect on incontinence status, but the sample recruited to the project was too small to provide definitive outcomes.

\section{Dose of intervention}

The main features of the Clinical Pathway were the early removal of catheters, prevention of dehydration and constipation, facilitation of toileting, and the identification, investigation and management of bladder symptoms. From the Patient Chart Audit, it seems that some of these actions were managed well (such as minimized duration of catheterization, and maintenance of fluids) when compared to a previous study (Chiarelli et al 2006), but some were not (such as bowel management). There were several important continence management actions that were either not carried out or not recorded on patient charts. Reviewing the results of the Chart Audit, it was apparent that the dose of intervention was not strong enough to make a difference to the care received by the intervention group compared with the control group.

Given that the PRN (a Registered Nurse) was not a member of Hospital Staff, but employed outside the system by the research project, they were specifically directed not to intervene clinically. The agreed protocol was that the PRN approach the Ward case managers or nursing staff to prompt continence management actions. However, this strategy was not compelling enough in the environment of competing demands that is the acute hospital setting. This lack of an "active agent" may have been a primary barrier to the implementation of the pathway.

It is recommended that for future projects aiming for research translation to practice in this setting, the agent for change should be from within the system the researcher is aiming to change. The advantages of this approach would be that the agent could clinically intervene, could refer to other providers as needed, and would already know the system well and the key people in the relevant disciplines which would ensure adequate communication with relevant staff. The agent's "intervention time" would add value to current practice by providing movement towards "best practice" with an efficient use of resources. These staff should be adequately backfilled so that the project can be enacted and implemented with maximum attention.

It was the intention of this study to embed the intervention within the normal hospital routine of the wards, to test if simple strategies could be effective in the "real world" setting of a busy hospital. The intervention was meant to be seamlessly integrated with patient care, so that patients not in the intervention group did not sense that their care was any different. This integration was successful and the intervention did occur around the normal day to day routine of staff, although the intervention itself was not powerful enough to show an effect on incontinence.

\section{Comparison with past studies}

There have been no past studies which have attempted to implement a continence promotion strategy for older people undergoing surgery for \#NOF. However, comparing the results for this pilot and a prior epidemiological survey conducted by this team (Chiarelli et al 2006), it appears that there may be a reversal of the worsening of symptoms observed in the epidemiological study postsurgery. Whereas patients in the epidemiological study demonstrated an increase in incontinence symptoms at five days post surgery and tended to maintain some higher levels at the 12-week follow-up, most participants in this pilot had fewer incontinence symptoms at 12 -weeks than before surgery. It is possible that some small global change occurred throughout the intervention, but such a conclusion cannot be confidently asserted due to the small sample size. 


\section{Some positive outcomes and recommendations}

Despite the non-significant results, there were some positive outcomes from the study. A well developed protocol now exists for recruitment in this target group, and a good understanding of expected accrual rates. A consent rate of $92 \%$ of eligible patients was achieved, demonstrating that the recruitment protocol was acceptable to this target group.

The case management model is available for replication. However it is recommended that future projects that aim for research translation to practice in this setting, should employ an agent for change from within the system. That is, the best model may be that the project "buys" some of the time of staff already employed within the system for them to act as intervention agents. Furthermore, the substantive area of responsibility of the agent should be as close as possible to intervention actions needed, where the best practice pathway can value add, and the project can value add by providing enough staff time for best practice actions to occur.

The evidence-based Clinical Pathway is in final form for this hospital, and provides a good basis for development of local Clinical Pathways in other hospitals. When introducing best practice, a good model for local adaptation is preferable to an inflexible fait a complit (NHS Centre for Reviews and Dissemination 1999).

The adaptation of the Clinical Pathway into a workable clinical tool, the B and B Checklist, was a positive outcome for the study. The B and B Checklist can be further adapted and simplified to ensure it can be easily integrated into the milieu of competing demands upon staff within the hospital system.

A continence promotion booklet specific to this target group ("Waterworks after Hip Surgery"), developed and piloted as an intervention resource, was both acceptable and useful to patients, and should be an integral component of strategies to manage incontinence for elderly patients undergoing surgery for \#NOF.

The \#NOF Newsletter was a good model for an additional communication strategy to ensure all staff were aware of the existence of, and progress of the study.

The evaluation tools for this study (five day survey, 12-week survey, chart audit tool, staff survey) have now been well-tested, and have been shown to be acceptable to the target group. The chart audit was a powerful tool for interpreting outcomes, and it should now be simplified to focus on the actions of most interest to the Clinical Pathway. Similarly, the staff survey was an effective tool for obtaining timely feedback from key collaborators.
A central outcome of this project was evidence that an intervention to reduce continence problems and improve continence management post surgery for older people in the acute hospital setting is imperative. However, the intervention implemented in this project included very broad-based strategies. More focused assessment and treatment for those most at risk of incontinence after \#NOF would be more acceptable to staff and a more efficient use of resources. A simple screening tool would ensure that those most at risk are detected, and targeted for care.

\section{Conclusion}

Given that the incidence of \#NOF is expected to double in the next 20-years (Sanders et al 1999), and the detrimental impact incontinence can have upon the health and wellbeing of older people (Fonda 1986; Grimby et al 1993; Millard 1996), it is important that effective interventions to detect those most at risk of incontinence and to promote continence after surgery for \#NOF are developed.

\section{Acknowledgments}

- Thanks to Marie Hiscox (Research Nurse), Jan McLeod (Research Interviewer), Ian Robinson (Statistical assistant), Dr Lynette Mackenzie (Occupational Therapist), and staff and patients of John Hunter Hospital and the (former) Royal Newcastle Hospital.

- This project was funded by the National Continence Management Strategy, an Australian Government Initiative.

- Researchers in the Faculty of Health at The University of Newcastle are members of Hunter Medical Research Institute (HMRI).

\section{References}

[AHCPR] American Agency for Healthcare Policy and Research. 1996. Clinical practice guidelines on urinary incontinence in adults. Rockville, Maryland: American Agency for Healthcare Policy and Research. US Department of Health and Human Services.

Boufous S, Finch C, Lord S. 2004. Incidence of hip fracture in NSW: are our efforts having an effect? Medical Journal of Australia, 180:623-6.

Charlson ME, Pompei P, Ales KL, et al. 1987. A new method of classifying prognostic comorbidity in longitudinal studies: development and validation. Journal of Chronic Diseases, 40:373-83.

Chiarelli P, Byles J, Parkinson L, et al. 2006. Changes in lower urinary tract symptoms following surgery for fractured neck of femur. Australian and New Zealand Continence Journal, 12:90-2.

Doak CC, Doak LG, Friedell GH, et al. 1998. Improving comprehension for cancer patients with low literacy skills: Strategies for Clinicians. Cancer Journal for Clinicians, 48:151-62.

Donovan J, Abrams P, Peters T, et al. 1996. The ICS-'BPH' Study: the psychometric validity and reliability of the ICS male questionnaire. British Journal of Urology, 77:554-62.

Doran C, Chiarelli P, Cockburn J. 2001. Economic costs of urinary incontinence in Community dwelling Australian women. Medical Journal of Australia, 174:456-8. 
Dray A. 1988. Epidural opiates and urinary retention: new models provide new insights. The Journal of Anesthesiology, 68:323-4.

Folstein M, Folstein S, McHugh P. 1975. Mini-mental State: a practical method of grading the cognitive state of patients for the clinician. Journal of Psychiatric Research, 12:189-98.

Fonda D. 1986. Urinary incontinence in the elderly. Its prevalence and cost implications. Australian Association of Gerontology Annual Conference, Melbourne.

Grimby A, Milsom I, Molander U, et al. 1993. The influence of urinary incontinence on the quality of life of elderly women. Age and Ageing, 22:82-9.

Gunthorpe W, Brown W, Redman S. 2000. The development and evaluation of an incontinence screening questionnaire for primary care. Neurology and Urodynamics, 19:597-607.

Hald T, Nordling J, Anderson J, et al. 1991. A patient weighted symptom score system in the evaluation of uncomplicated benign prostatic hyperplasia. Scandinavian Journal of Urology and Nephrology Suppl, 138:59-62.

Halm EA, Magaziner J, Hannan EL, et al. 2003. Frequency and impact of active clinical issues and new impairments on hospital discharge in patients with hip fracture. Archives of Internal Medicine, 163:108-13.

[HSRG] Health Services Research Group. 2006. NSW Department of Health Inpatients Statistics Collection (ISC) data 2003-2004. Health Services Research Group Summary, CCEB, University of Newcastle.

Johansson I, Athlin E, Frykholm L, et al. 2002. Intermittent versus indwelling catheters for older patients with hip fractures. Journal of Clinical Nursing, 11:651-6.

Kitchiner D, Bundred PE. 1999. Clinical Pathways: a practical tool for specifying, evaluating and improving the quality of clinical practice. Medical Journal of Australia, 170:54-5.

Kreuter MW, Farrell D, Olevitch L, et al. 2000. Tailoring health messages: Customising communication with computer technology. Mahwah, NJ: Lawrence Erlbaum.

March L, Chamberlain A, Cameron I, et al. 1996. Prevention, treatment and rehabilitation of fractured neck of femur. Report from the Northern Sydney Area Fractured Neck of Femur Health Outcomes Project. To NSW Health - Health Outcomes Program Grant Scheme 1994-1996, Sydney.
Millard RJ. 1996. Urinary incontinence: the Cinderella subject. Medical Journal of Australia, 165:124-5.

Meigs JB, Barry MJ, Giovannucci E, et al. 1999. Incidence rates and risk factors for acute urinary retention: the health professionals follow-up study. The Journal of Urology, 162:376-82.

NHS Centre for Reviews and Dissemination. 1999. Getting evidence into practice. Effective Health Care, 5(1).

Ouslander JG. 1990. Urinary incontinence in nursing homes. Journal of the American Gerontological Association, 38:289-91.

Palmer M, Myers A, Fedenko K. 1997. Urinary continence changes after hip fracture repair. Clinical Nursing Research, 6:8-24.

Petterson T. 1994. How readable are the hospital information leaflets available to elderly patients? Age and Ageing, 23:14-6.

Ringdal M, Borg B, Hellstrom A. 2003. A survey on incidence and factors that may influence first postoperative urination. Urologic Nursing, 23:341-5.

Ryan P, Lauver R. 2002. The efficacy of tailored interventions. Journal of Nursing Scholarship, 34:331-7.

Sanders KM, Nicholson G, Ugoni A, et al. 1999. Health burden of hip and other fractures in Australia beyond 2000. Projections based on the Geelong Osteoporosis Study. Medical Journal of Australia, 170:467-70.

Sandvik H, Hunskaar S, Seim A, et al. 1993. Validation of a severity index in female urinary incontinence and its implementation in an epidemiological survey. Journal of Epidemiology and Community Health, 47:497-9.

Skelly JM, Guyatt GH, Kalbfleisch R, et al. 1992. Management of urinary retention after surgical repair of hip fracture. Canadian Medical Association Journal, 146:1185-9.

Smith W. \$15 Million for National Continence Management Strategy. http://www.health.gov.au/archive/mediarel/1998/ws4498.htm. t. $J$ Media Release.

Thom D, Haan M, Eeden SVD. 1997. Medically recognized urinary incontinence and risks of hospitalisation, nursing home admission and mortality. Age and Ageing, 26:367-74.

Watts W, Lloyd G, Brown W, et al. 2002. Routine clean intermittent selfcatheterisation: Innovative implementation in the Hunter Area Health Service. Urologic Nursing, 22:119-23. 